Si mil at $i$ on of $f$ ast- $i$ on- $d r i$ ven $A l f$ ven ei genmodes on the Experi ment al Advanced Super conduct i ng Tokamak 


\title{
Simulation of fast-ion-driven Alfvén eigenmodes on the Experimental Advanced Superconducting Tokamak
}

\author{
Youjun $\mathrm{Hu}^{\mathrm{a}},{ }^{1,2}$ Y. Todo, ${ }^{3}$ Youbin Pei,${ }^{1}$ Guoqiang Li, ${ }^{1}$ Jinping Qian, ${ }^{1}$ \\ Nong Xiang, ${ }^{1}$ Deng Zhou, ${ }^{1}$ Qilong Ren, ${ }^{1}$ Juan Huang, ${ }^{1}$ and Liqing Xu ${ }^{1}$ \\ ${ }^{1}$ Institute of Plasma Physics, Chinese Academy \\ of Sciences, Hefei, Anhui 230031, China \\ ${ }^{2}$ Center for Magnetic Fusion Theory, \\ Chinese Academy of Sciences, Hefei, Anhui 230031, China \\ ${ }^{3}$ National Institute for Fusion Science, Toki, Gifu 509-5292, Japan
}

\begin{abstract}
Kinetic-MHD hybrid simulations are carried out to investigate possible fast-ion-driven modes on the Experimental Advanced Superconducting Tokamak (EAST). Three typical kinds of fast-ion-driven modes, namely Toroidicity-induced Alfvén Eigenmodes (TAEs), Reversed Shear Alfvén Eigenmodes (RSAEs), and Energetic-Particle continuum Modes (EPMs), are observed simultaneously in the simulations. The simulation results are compared with the results of an ideal MHD eigenvalue code, which shows agreement with respect to the mode frequency, dominant poloidal mode numbers, and radial location. However, the modes in the hybrid simulations take a twisted structure on the poloidal plane, which is different from the results of the ideal MHD eigenvalue code. The twist is due to the radial phase variation of the eigenfunction, which may be attributed to the non-perturbative kinetic effects of the fast ions. By varying the stored energy of fast ions to change the fast ion drive in the simulations, it is demonstrated that the twist (i.e., the radial phase variation) is positively correlated with the fast ion drive.
\end{abstract}

a E-mail: yjhu@ipp.cas.cn 


\section{INTRODUCTION}

Alfvén eigenmodes (AEs) can be excited in tokamak plasmas by fast ions from various sources including neutral beam injection (NBI), RF heating, and fusion reactions[1-8]. The electromagnetic fluctuations of the AEs have the possibility of influencing the transport of fast ions and thus are important in determining the performance of NBI/RF heating and future burning plasmas[9-17]. The Experimental Advanced Superconducting Tokamak (EAST)[18] has recently been upgraded to include four deuterium neutral beam lines. Figure 1 shows the beam injection geometry on EAST. It is expected that, with the NBI generated fast ions, various AEs will be routinely observed on EAST. In the present work, kinetic-MHD hybrid simulations using MEGA code[19-21] are carried out to investigate possible fast-ion-driven Alfvén eigenmodes on EAST. The simulations use an equilibrium with a flat profile of safety factor. Anisotropic slowing down distributions are used to model the distribution of the fast ions from the neutral beam injection. Perturbations of multiple toroidal mode numbers are included in the simulations. Plenty of modes in the Alfvén frequency range are found in the simulation. The frequency and radial width of the modes observed in the simulations are compared with the Alfvén continua to categorize the modes, which indicates the modes include the Toroidicity-induced Alfvén Eigenmodes (TAEs)[1, 3, 4, 19, 22], Reversed Shear Alfvén Eigenmodes (RSAEs)[7, 8, 23-31], and Energetic-Particle continuum Modes (EPMs) $[5,20,32,33]$. The simulation results are also compared with the results of a MHD eigenvalue code[34], which shows agreement with respect to the frequency, dominant poloidal mode number, and radial location of the modes.

Measurements from the electron cyclotron emission radiometer show that the phase of AEs usually changes across the radius[35-37]. The radial phase variation makes the two-dimensional mode structure on the poloidal plane take a twisted 


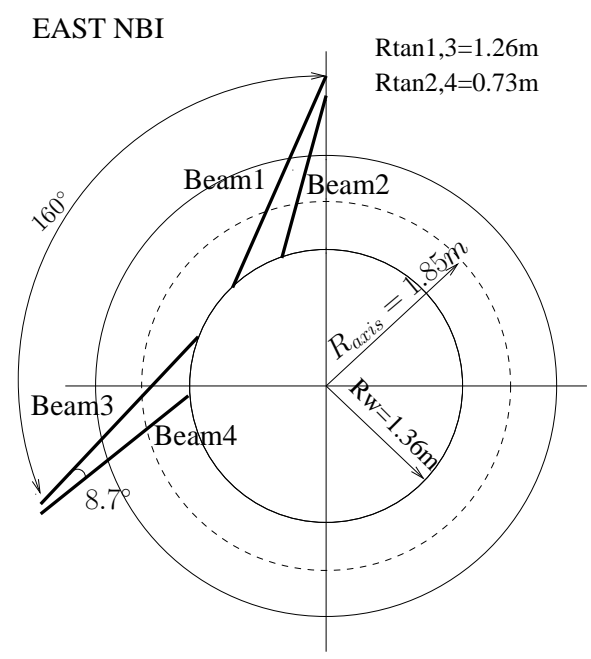

Figure 1. Top view of the neutral beam injection geometry on EAST tokamak. The four beams all lie on the midplane of the device with the tangential radii of the beams being $R_{\tan 1}=R_{\tan 3}=1.26 m$ and $R_{\tan 2}=R_{\tan 4}=0.73 m$ (the tangential radius of a beam line is the perpendicular distance of the beam line to the axisymmetric axis of the device). The maximum source power per beam line is $2 \mathrm{MW}$. The maximum injection energy of particles is $80 \mathrm{keV}$.

structure. The cause of the twist (or radial phase variation) is usually attributed to the non-perturbative kinetic effects of energetic particles[38-40]. In the present hybrid simulations, two-dimensional mode twist on the poloidal plane is observed, which is different from the results of the ideal MHD eigenvalue code. By varying the stored energy of fast ions to change the fast ions drive in the simulations, the dependence of the radial phase variation on the energetic particles drive is examined, which indicates that the radial phase variation is positively correlated with the fast ions drive.

The remainder of this paper is organized as follows. Section II reviews the model used in the simulation code MEGA. Section III gives the equilibrium used in the 
simulation. The simulation results are given in Sec. IV. A brief summary is given in Sec. V.

\section{SIMULATION MODEL}

MEGA is a numerical code calculating the interaction of thermal plasmas and energetic particles (EPs) in toroidal geometries[20]. In MEGA, the thermal plasmas are described by the nonlinear full MHD equations while the EPs are described by the drift-kinetic equation. The MHD equations solved by MEGA consist of the mass continuum equation

$$
\frac{\partial \rho}{\partial t}=-\nabla \cdot(\rho \mathbf{u})+\nu_{n} \nabla^{2}\left(\rho-\rho_{\mathrm{eq}}\right)
$$

the momentum equation (with modification by the effects of EPs)

$$
\rho \frac{\partial \mathbf{u}}{\partial t}=-\rho \boldsymbol{\Omega} \times \mathbf{u}-\rho \nabla\left(\frac{u^{2}}{2}\right)-\nabla p+\left(\mathbf{j}-\mathbf{j}_{h}^{\prime}\right) \times \mathbf{B}+\frac{4}{3} \nabla(\nu \rho \nabla \cdot \mathbf{u})-\nabla \times(\nu \rho \boldsymbol{\Omega}),
$$

the Faraday's law

$$
\frac{\partial \mathbf{B}}{\partial t}=-\nabla \times \mathbf{E}
$$

and the equation of state

$\frac{\partial p}{\partial t}=-\nabla \cdot(p \mathbf{u})-(\Gamma-1) p \nabla \cdot \mathbf{u}+(\Gamma-1)\left[\nu \rho \Omega^{2}+\frac{4}{3} \nu \rho(\nabla \cdot \mathbf{u})^{2}+\eta \mathbf{j} \cdot\left(\mathbf{j}-\mathbf{j}_{\text {eq }}\right)\right]+\nu_{n} \nabla^{2}\left(p-p_{\text {eq }}\right)$,

where $\rho, \mathbf{u}$, and $p$ are the mass density, fluid velocity, and pressure of the thermal plasma, respectively, $\mathbf{B}$ and $\mathbf{E}$ are the magnetic field and electric field, the electric field $\mathbf{E}$ is given by $\mathbf{E}=-\mathbf{u} \times \mathbf{B}+\eta\left(\mathbf{j}-\mathbf{j}_{\text {eq }}\right)$, the current density $\mathbf{j}$ is given by $\mathbf{j}=\mu_{0}^{-1} \nabla \times \mathbf{B}$, the vorticity $\Omega$ is given by $\Omega=\nabla \times \mathbf{u}, \mu_{0}$ is the vacuum magnetic permeability, $\Gamma$ is the adiabatic constant $(\Gamma=5 / 3$ in the simulations presented here), $\eta$ is the electric resistivity, $\nu$ and $\nu_{n}$ are the artificial viscosity and diffusion coefficients chosen to maintain numerical stability (these dissipation coefficients also 
play a physical role of enhancing the damping of the modes in the MHD simulation that does not include kinetic damping from the thermal plasma), the subscript "eq" represents the equilibrium variables, $\mathbf{j}_{h}^{\prime}$ is the current density of EPs without the contribution of $\mathbf{E} \times \mathbf{B}$ drift (the contribution of $\mathbf{E} \times \mathbf{B}$ drift disappears due to quasineutrality[19]). Note that the effects of EPs on thermal plasma enter through the $\mathbf{j}_{h}^{\prime}$ term in the momentum equation (2) of the thermal plasma. This scheme of coupling EPs with thermal plasma (usually called "current coupling") is valid when the density of EPs is low so that the the inertia of EPs can be neglected in the momentum equation.

In MEGA, the MHD equations (1)-(4) are discretized in space by using a fourthorder finite difference scheme in the right-handed cylindrical coordinates $(R, \phi, Z)$. The time discretization uses the fourth-order Runge-Kutta method.

The drift-kinetic description is employed for the EPs. The guiding-center motion of EPs are governed by the following equations

$$
\begin{gathered}
\frac{d \mathbf{X}}{d t}=\frac{\mathbf{B}^{\star}}{B_{\|}^{\star}} v_{\|}+\mathbf{v}_{\nabla B}+\mathbf{v}_{E \times B} \\
\frac{d v_{\|}}{d t}=-\frac{\mu}{m_{h}} \frac{\mathbf{B}^{\star}}{B_{\|}^{\star}} \cdot \nabla B+\frac{Z_{h} e}{m_{h}} \frac{\mathbf{B}^{\star}}{B_{\|}^{\star}} \cdot \mathbf{E},
\end{gathered}
$$

where $\mathbf{X}$ is the location of the guiding-centers, $v_{\|}$is the parallel (to the magnetic field) velocity, $\mathbf{v}_{\nabla B}$ is the $\nabla B$ drift given by $\mathbf{v}_{\nabla B}=\mu \mathbf{B} \times \nabla B /\left(m_{h} \Omega_{h} B_{\|}^{\star}\right), \mathbf{v}_{E \times B}$ is the $\mathbf{E} \times \mathbf{B}$ drift given by $\mathbf{v}_{E \times B}=\mathbf{E} \times \mathbf{B} /\left(B B_{\|}^{\star}\right), \mu$ is the magnetic moment, $m_{h}, Z_{h} e$, and $\Omega_{h}$ are the mass, electric charge, cyclotron angular frequency of EPs, respectively; $\mathbf{B}^{\star}$ and $B_{\|}^{\star}$ are defined by

$$
\begin{gathered}
\mathbf{B}^{\star}=\mathbf{B}+B \frac{v_{\|}}{\Omega_{h}} \nabla \times \mathbf{b}, \\
B_{\|}^{\star} \equiv \mathbf{b} \cdot \mathbf{B}^{\star}=B\left(1+\frac{v_{\|}}{\Omega_{h}} \mathbf{b} \cdot \nabla \times \mathbf{b}\right),
\end{gathered}
$$


respectively, where $\mathbf{b}=\mathbf{B} / B$. In MEGA, the orbit of the EP guiding-centers are followed by using the fourth-order Runge-Kutta method. Expressed in terms of the guiding-center drift, the current density $\mathbf{j}_{h}^{\prime}$ appearing in Eq. (2) is written

$$
\mathbf{j}_{h}^{\prime}=\int f\left(\frac{\mathbf{B}^{\star}}{B_{\|}^{\star}} v_{\|}+\mathbf{v}_{\nabla B}\right) Z_{h} e d^{3} v-\nabla \times \int f \mu \mathbf{b} d^{3} v
$$

where $f$ is the guiding-center distribution function of EPs, the last term on the righthand side is the magnetization current. Note again that the $\mathbf{E} \times \mathbf{B}$ drift does not appear in Eq. (9) due to the quasi-neutrality[19]. In MEGA, the evolution of the distribution function of EPs is simulated by using the $\delta f$ particle-in-cell method.

\section{EQUILIBRIUM}

The EAST equilibrium used here was reconstructed by the EFIT code[41] by using the constraints from experimental diagnostics in EAST discharge \#48916 at 4.5s. Figure 2 plots the flux surfaces of the equilibrium and the computational box on the poloidal plane used in the simulation. The computational box is chosen to enclose the flux surface with $\sqrt{\bar{\Psi}_{t}}=99 \%$, where $\sqrt{\bar{\Psi}_{t}}$ is the square root of the normalized toroidal magnetic flux.

The profiles of the safety factor $q$, plasma pressure $p_{0}$, and electron number density $n_{e 0}$ of the equilibrium are plotted in Fig. 3. The profile of the safety factor has a weak negative shear in the region $\sqrt{\bar{\Psi}_{t}} \leqslant 0.4$. The electron number density $n_{e 0}$ is used here to determine the mass density of the thermal Deuterium plasma through the approximate relation $\rho_{0} \approx n_{e 0} m_{i}$, where $m_{i}$ is the mass of Deuteron.

In this work, the equilibrium distribution of the fast ions from the Deuterium neutral beam injection is modeled by the anisotropic slowing down distributions, 


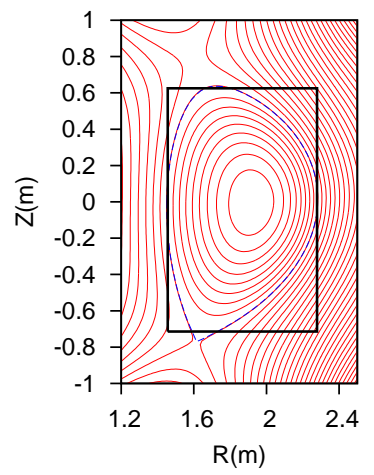

Figure 2. Flux surfaces shape of EAST discharge \#48916 at 4.5s. The black rectangle indicates the computational box on the poloidal plane used in the simulation. The magnetic field at the magnetic axis $B_{\varphi 0}=+1.72$ Tesla, the toroidal plasma current $I_{p \phi}=-417 \mathrm{kA}$.

which takes the following form

$$
f_{\text {eq }}(\psi, v, \lambda)=C \exp \left(-\frac{\psi_{p}}{\psi_{\text {scale }}}\right) \frac{1}{v^{3}+v_{\text {crit }}^{3}} \frac{1}{2} \operatorname{erfc}\left(\frac{v-v_{b}}{\Delta_{v}}\right) \exp \left(-\frac{\left(\lambda-\lambda_{0}\right)^{2}}{\Delta_{\lambda}^{2}}\right),
$$

where $C$ is a constant, which is chosen to achieve desired stored energy of fast ions; $\psi_{p}$ is the normalized poloidal flux, $\psi_{\text {scale }}$ is a quantity characterizing the radial gradient, $v$ is the velocity of fast ions, $v_{b}$ is the injection velocity of the neutral beam, $\Delta_{v}$ is a small velocity (compared with $v_{b}$ ), which is used to set the cutoff width near $v_{b} ; \lambda$ is the the normalized magnetic moment defined by $\lambda=\mu B_{0} / \varepsilon$, where $B_{0}$ is the strength of the equilibrium magnetic field at the magnetic axis, $\varepsilon$ is the kinetic energy of fast ions, $\lambda_{0}$ and $\Delta_{\lambda}$ characterize the peak location and the width of the distribution over the pitch angle, $v_{\text {crit }}$ is the critical velocity for the collisional friction of fast ions with thermal electrons and ions being equal, which is given by[42]

$$
v_{\text {crit }}=\left(\frac{m_{e}}{m_{i}} \frac{3 \sqrt{\pi}}{4}\right)^{1 / 3} v_{\text {te }}
$$

where $m_{e}$ and $v_{t e}$ are the mass and thermal velocity of the electrons. In this work the beam velocity is chosen $v_{b}=2.35 \times 10^{6} \mathrm{~m} / \mathrm{s}$, which is the velocity of a Deuteron 

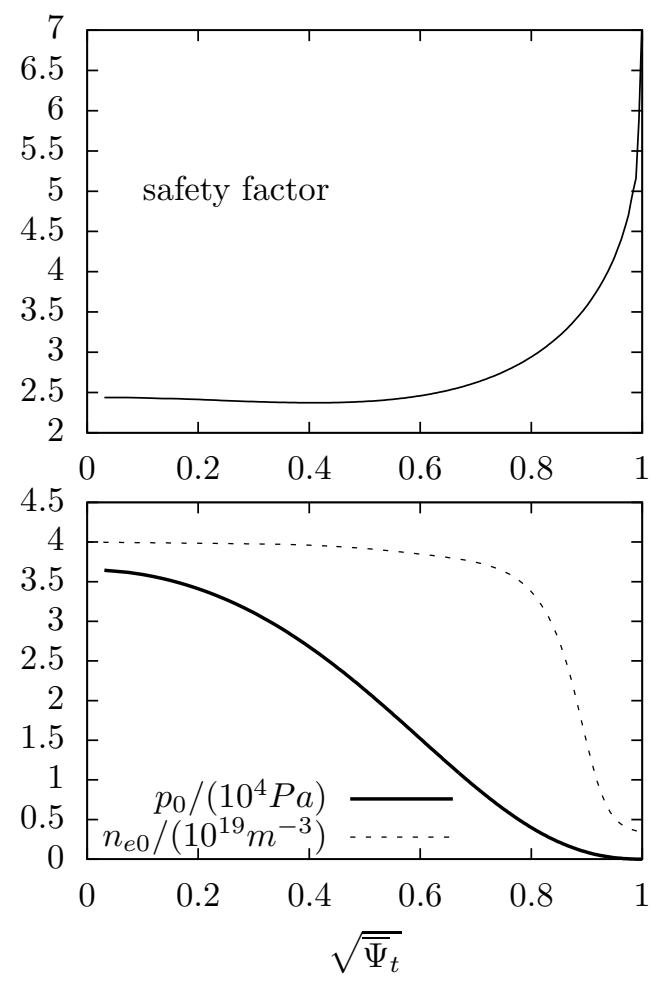

Figure 3. The radial profiles of the safety factor, plasma pressure, and electron number density for EAST discharge \#48916 at 4.5s. The profile of the safety factor is flat and has a weak negative shear in the region $\sqrt{\bar{\Psi}_{t}} \leqslant 0.4$ (the safety factor reaches its minimum value $q_{\min }=2.373$ at $\left.\sqrt{\bar{\Psi}_{t}}=0.40\right)$. The value of the safety factor at the magnetic axis $q_{0}=2.438$. The value of the safety factor at the flux surface enclosing $95 \%$ of the poloidal magnetic flux $q_{95}=4.274$. The stored plasma energy of the equilibrium is $127 \mathrm{~kJ}$.

with kinetic energy of $58 \mathrm{keV} ; v_{\text {crit }}=1.89 \times 10^{6} \mathrm{~m} / \mathrm{s}$, which corresponds to the critical velocity given by Eq. (11) evaluated with the electron temperature $T_{e}=2 \mathrm{keV}$. The small cutoff width near the beam velocity is chosen as $\Delta_{v}=0.21 \times 10^{6} \mathrm{~m} / \mathrm{s}$. The central pitch angle variable $\lambda_{0}$ is chosen as $\lambda_{0}=0.5$ with the expansion width $\Delta_{\lambda}$ 
chosen as $\Delta_{\lambda}=0.3$, which represents a reasonable distribution over the pitch angle based on the beam injection geometry on EAST. The parameter $\psi_{\text {scale }}$, characterizing the radial gradient of the fast ion pressure, is chosen as $\psi_{\text {scale }}=0.4$.

The pressure $p_{0}$ given in Fig. 3 is the total pressure, which should include the contributions from both the thermal plasma and fast ions. However, in the present work, for simplicity, the pressure $p_{0}$ given in Fig. 3 is assumed to be the pressure of the thermal plasma.

\section{SIMULATION RESULTS}

In the simulations, all the MHD perturbations are set to be zero at the computational boundary. Initial perturbation within the computational boundary is set to be random magnetic perturbation of order $\delta B / B_{0} \approx 10^{-10}$ (via the curl of a random vector field, which ensures the divergence-free nature of the magnetic perturbation). The numbers of grid points for cylindrical coordinates $(R, \phi, Z)$ are $(128,64,128)$. The number of particle markers loaded is $2^{20}$, which approximately corresponds to one marker per spacial grid. To reduce numerical noise, the MHD perturbations are filtered to keep only harmonics of low toroidal mode numbers. In this work, harmonics out of the range $-8 \leqslant n \leqslant 8$ are filtered out every 1000 time steps, where $n$ is the toroidal mode number. The time step $d t$ is chosen to be $d t=0.1 / \Omega_{h 0}=1.2 \times 10^{-9} s$, where $\Omega_{h 0}$ is the cyclotron angular frequency of the fast ions at the magnetic axis. This small time step (compared with the cyclotron period of fast ions) is chosen to meet the Courant condition of the full MHD system (for the present case, the Courant number $d t V_{\max } / d x \approx 0.9<1$, where $V_{\max }$ is the maximum of the Alfvén speed in the computational region, $d x$ is one of the spacial grid intervals). The ratio of the time step $d t$ to the Alfvén time $t_{A}$ is $d t / t_{A}=2.65 \times 10^{-3}$, where $t_{A}$ is defined by $t_{A}=R_{\text {axis }} / V_{A 0}$ with $R_{\text {axis }}$ being the major radius of the magnetic axis and $V_{A 0}$ 
being the Alfvén speed at the magnetic axis. In MEGA, the fluid velocity $\mathbf{u}$ is normalized by $V_{A 0}$. In the simulations, the artificial viscosity and diffusion coefficients are chosen as $\nu=\nu_{n}=1.0 \times 10^{-7} V_{A 0} R_{\text {axis. }}$. The electric resistivity is chosen as $\eta=1.0 \times 10^{-7} \mu_{0} V_{A 0} R_{\text {axis }}$.

Although MEGA code uses cylindrical coordinates $(R, \phi, Z)$ in advancing the MHD equations and the orbit of energetic particles, it uses flux coordinates $(\psi, \theta, \phi)$ in analyzing the simulation results, where $\psi$ is a magnetic surface label (in this article, $\psi$ is chosen as $\left.\psi=\sqrt{\bar{\Psi}_{t}}\right), \phi$ is the usual toroidal angle, $\theta$ is chosen to make magnetic field lines straight on $(\theta, \phi)$ plane (the transformation Jacobian of the coordinates is proportional to $h(\psi) R^{2}$, where $h(\psi)$ is a flux function). The zero point of $\theta$ coordinate is chosen at the low-field side of the midplane and the positive direction of $\theta$ is chosen in the counterclockwise direction when viewed in $\hat{\phi}$ direction. In $(\psi, \theta, \phi)$ coordinates system, the radial component of the fluid velocity $u_{\psi}$ can be expanded in two-dimensional Fourier series over $\theta$ and $\phi$, which is written

$$
u_{\psi}(\psi, \theta, \phi, t)=\sum_{n=-\infty}^{\infty} \sum_{m=0}^{\infty}\left[u_{\psi m n}^{(c)}(\psi, t) \cos (m \theta+n \phi)+u_{\psi m n}^{(s)}(\psi, t) \sin (m \theta+n \phi)\right]
$$

where $u_{\psi m n}^{(c)}(\psi, t)$ and $u_{\psi m n}^{(s)}(\psi, t)$ are cosine and sine components of the expansion coefficients, respectively. Note that, in this expansion, the poloidal mode number $m$ is always positive while the toroidal mode number $n$ can be negative.

Figure 4 plots the two-dimensional mode structure observed in the simulation at time slice $t=0.25 \mathrm{~ms}$, which shows that the $n=-8$ mode has the largest amplitude. This mode localizes in a narrow radial region and takes a ballooning structure with a large dominant poloidal mode number $(m=24)$, as is shown in Figure 4h. Furthermore, after examining the temporal evolution of the $n=-8$ mode, we found it is a purely growing mode with zero frequency. Considering this, this mode is identified as a MHD ballooning mode, which will not be discussed further in this paper. Similarly the $n=-7$ mode is also a MHD ballooning mode. 
Excluding these two modes, the dominant modes among the modes given in Fig. 4 are the $n=-2, n=-3$, and $n=-4$ modes, which will be analyzed in turn next.
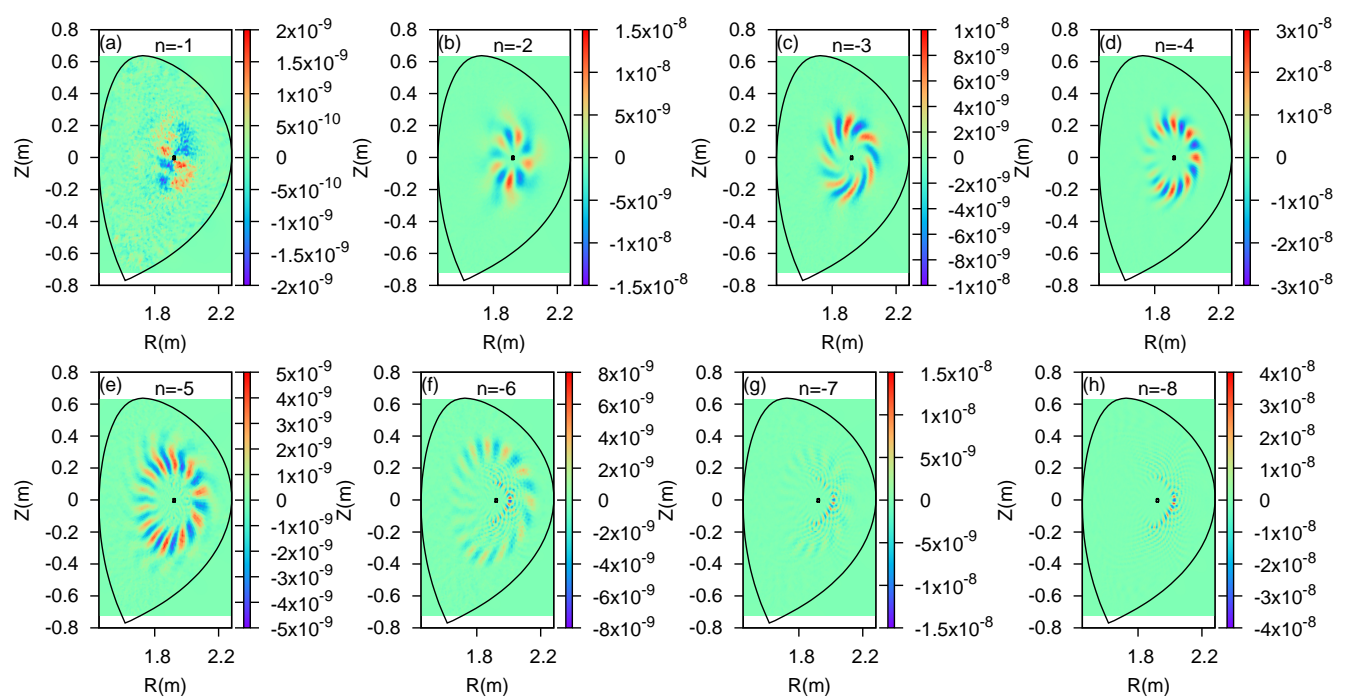

Figure 4. Contour of the toroidal harmonics of the radial velocity $u_{\psi}$ on the poloidal plane for different toroidal mode number $n$ (the toroidal mode numbers are indicated on the corresponding figures) at time $t=0.25 \mathrm{~ms}$. The initial stored energy of the fast ions within the boundary flux surface is $35 \mathrm{~kJ}$ for this case. The back line on every figure indicates the last closed flux surface.

\section{A. Identification of the fast-ion-driven modes}

The radial structures of the various poloidal harmonics of the $n=-2$ modes are plotted in Fig. 5, which shows that the $m=5$ harmonics is dominant and the mode amplitude reaches its peak at the radial location $\sqrt{\bar{\Psi}_{t}}=0.2$. 

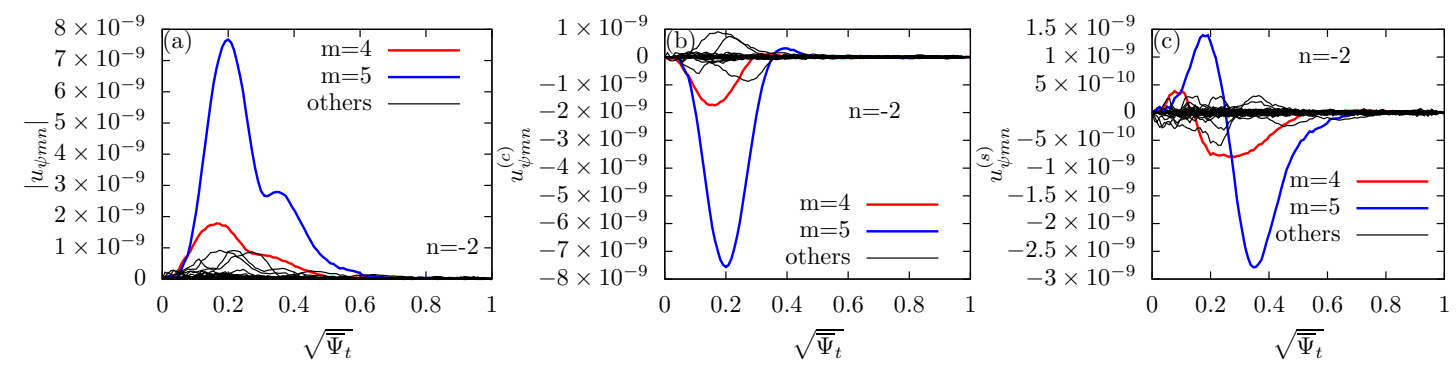

Figure 5. The radial profiles of (a) amplitude $\left|u_{\psi m n}\right|$, (b) cosine components $u_{\psi m n}^{(c)}$, and (c) sine components $u_{\psi m n}^{(s)}$ of the $n=-2$ mode. The time for plotting these figures is chosen to be at the moment when the sine component of the dominant $m=5$ harmonic reaches zero at the radial location where the amplitude of the mode is maximal (i.e., $\sqrt{\bar{\Psi}_{t}}=0.2$ for this case). The poloidal mode number range for this case is $0 \leqslant m \leqslant 64$.

A general harmonic in the Fourier expansion (12) can be further written as

$$
u_{\psi m n}^{(c)}(\psi, t) \cos (m \theta+n \phi)+u_{\psi m n}^{(s)}(\psi, t) \sin (m \theta+n \phi)=A \cos [\alpha-(m \theta+n \phi)],
$$

with the amplitude $A$ given by $A=\left|u_{\psi m n}\right|=\sqrt{\left(u_{\psi m n}^{(c)}\right)^{2}+\left(u_{\psi m n}^{(s)}\right)^{2}}$ and the phase angle $\alpha$ given by $\alpha=\operatorname{atan}\left(u_{\psi m n}^{(s)}, u_{\psi m n}^{(c)}\right)$, where $\operatorname{atan}()$ is an arc tangent function with two arguments that can determine the correct quadrant of the results. Using this, the instantaneous angular frequency of a harmonic is obtained by calculating the temporal change rate of the phase angle $\alpha$, i.e., $\omega=d \alpha / d t$. The growth rate is given by $\gamma=d A / d t$. The frequency and growth rate of the $n=-2$ mode can be obtained by calculating the frequency and growth rate of the dominant poloidal harmonic at the radial location where the amplitude of the mode is maximal. Figure 6 plots the temporal evolution of the $m=5$ poloidal harmonic at the radial location $\sqrt{\bar{\Psi}_{t}}=0.2$. The results show that the frequency of the mode $\omega / 2 \pi=69.0 \mathrm{kHz}$ and the linear growth rate $\gamma=2.71 \times 10^{4} s^{-1}$.

Using the formula in Eq. (13) and considering the sign of the mode numbers 
$(m, n)$, the sign of the frequency, and the defined positive directions of the poloidal angle $\theta$ and toroidal angle $\phi$, we can determine the toroidal and poloidal propagation directions of the $n=-2$ mode. The mode propagates poloidally in $+\hat{\boldsymbol{\theta}}$ direction and toroidally in $-\hat{\phi}$ direction. In terms of the physical quantities, the mode propagate poloidally in the diamagentic drift direction of the ions and toroidally in the cocurrent direction, which is consistent with the general rules for the propagation direction of Alfvén modes excited by fast ions[43].
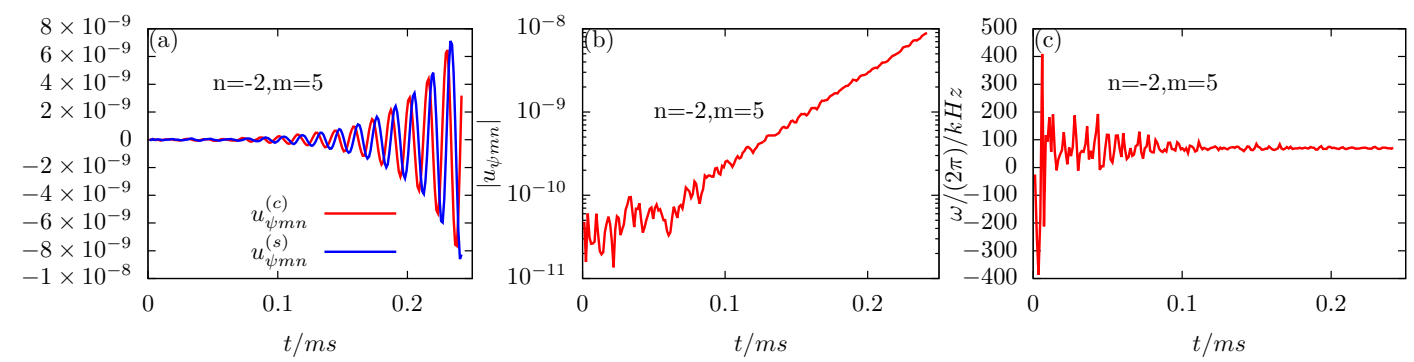

Figure 6. Temporal evolution of (a) the cosine component $u_{\psi m n}^{(c)}$ and sine component $u_{\psi m n}^{(s)}$, (b) the amplitude $\left|u_{\psi m n}\right|$ (in logarithm scale), and (c) the frequency of the $m=5$ harmonic of the $n=-2$ mode. The linear growth rate calculated from these data is $\gamma=2.71 \times 10^{4} s^{-1}$. A more convenient way to calculate the frequency (without sign) is to calculate the time interval for the oscillation $\left(u_{\psi m n}^{(c)}\right.$ or $\left.u_{\psi m n}^{(s)}\right)$ reaching two successive zero points to get the period of the oscillation and thus the frequency, which gives $\omega / 2 \pi=69.0 \mathrm{kHZ}$.

To identify which kind of fast-ions driven mode the $n=-2$ mode discussed above belongs to, we plot the frequency and the Half Height Full Width (HHFW) of the mode on the graphic of the Alfvén continua, as shown in Fig. 7, which indicates that the mode lies in the TAE gap. Also plotted on Fig. $7 \mathrm{a}$ is the $m=4$ and $m=5$ Alfvén continua in the cylindrical limit, which shows that the two continua are well separated from each other, indicating there will be only weak coupling between these two harmonics in the corresponding toroidal geometry. Furthermore, as shown in Fig. 
5 , the mode is dominated by the $m=5$ harmonic with the $m=4$ harmonic being much smaller, thus excluding the possibility of being an TAE mode. Also note that the radial location of the mode is near the location where the safety factor reaches its minimal value $\left(\sqrt{\bar{\Psi}_{t}}=0.4\right)$. Considering these characteristics of the mode, it is reasonable to identify the mode as a RSAE.
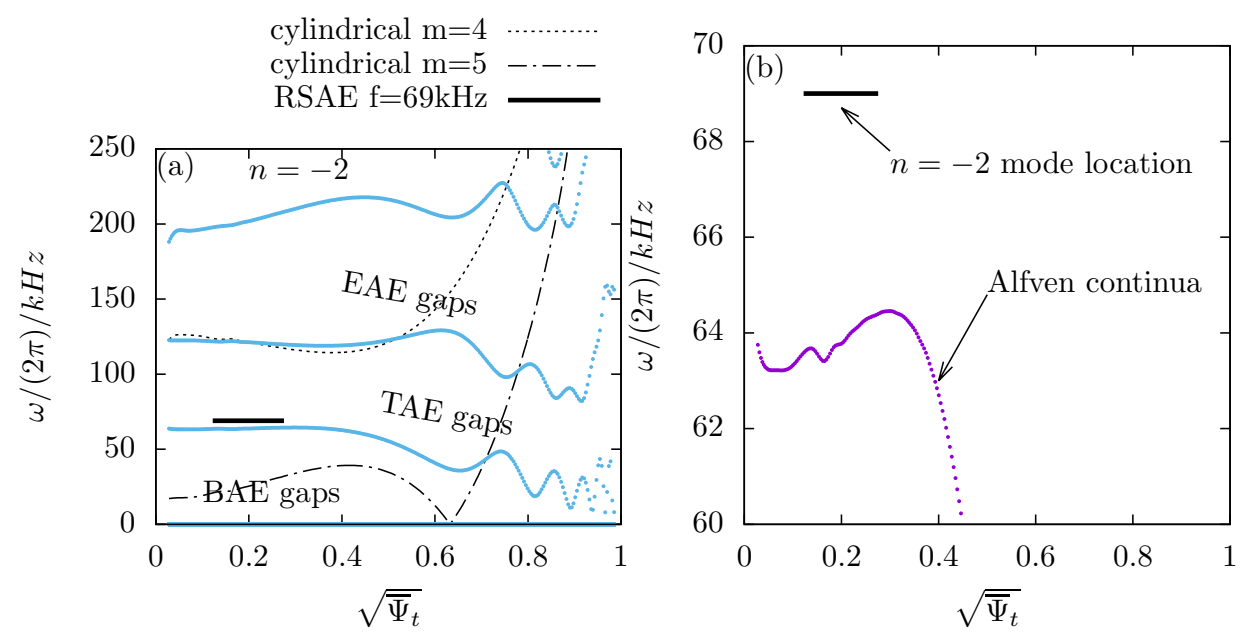

Figure 7. (a) $n=-2$ Alfvén continua with the frequency and the HHFW of the $n=-2$ mode plotted on. Also plotted on (a) are the $m=4$ and $m=5$ Alfvén continua in the cylindrical geometry limit. (b) Enlarged part of the Alfvén continua in the frequency range $[60 \mathrm{kHz}: 70 \mathrm{kHz}]$ to show the tip of the continua near the zero magnetic shear point. The Alfvén continua is calculated in the slow-sound approximation[44, 45].

Similar analysis can be applied to the $n=-3$ mode. The results are plotted in Figs. 8 and 9 . Figure 8 shows the dominant poloidal mode number is $m=7$ and all other poloidal harmonics are negligible. The frequency of the the $n=-3$ mode is $\omega / 2 \pi=69.0 \mathrm{kHz}$, which happens to be identical to the frequency of the $n=-2$ RSAE analyzed above. Figure 9 plots the frequency and the half height full width of the dominant $m=7$ harmonics on the graphic of the Alfvén continua, which 
indicates the mode intersect with the $m=7$ Alfvén continua. Considering these characteristics of the mode, it is reasonable to identify the mode as an EnergeticParticle continuum Mode (EPM), instead of a gap mode.
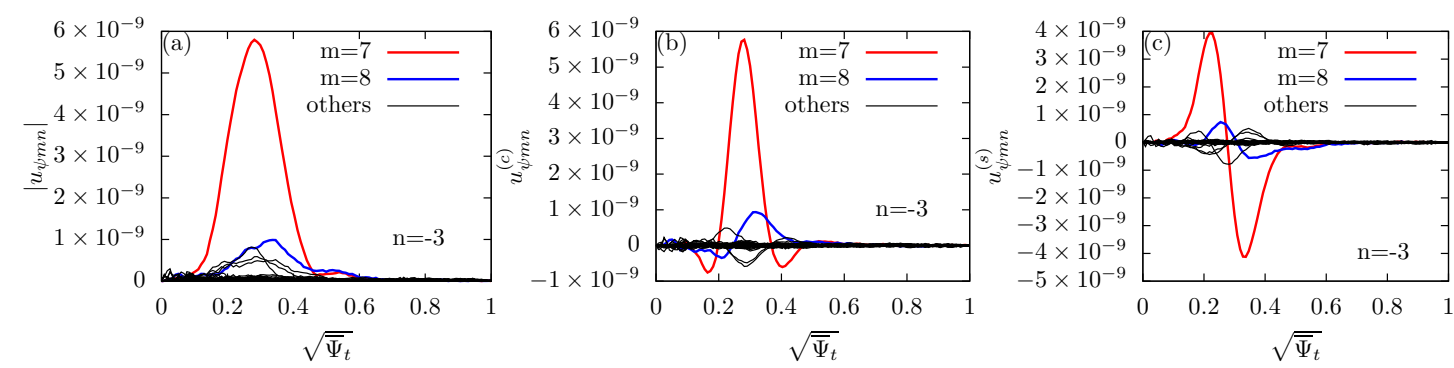

Figure 8. The radial profiles of (a) the amplitude $\left|u_{\psi m n}\right|$, (b) cosine components $u_{\psi m n}^{(c)}$, and (c) sine components $u_{\psi m n}^{(s)}$ of the $n=-3$ mode. The time for plotting these figures is chosen to be at the moment when the sine component of the dominant $m=7$ harmonic reaches zero at the radial location where the amplitude of the mode is maximal (i.e., $\sqrt{\bar{\Psi}_{t}}=0.28$ for this case). The poloidal mode number range for this case is $0 \leqslant m \leqslant 64$.

Similar analysis can also be applied to the $n=-4$ mode. The results are plotted in Figs. 10 and 11. Figure 10 shows that there are two dominant harmonics for this case, namely $m=9$ and $m=10$. The frequency of the mode is $\omega / 2 \pi=82.8 \mathrm{kHz}$. Figure 11 plots the frequency and the HHFW of the dominant $m=10$ harmonic on the graphic of the MHD continua, which indicates the mode lies in the TAE gap formed due to the coupling of the $m=9$ and $m=10$ harmonics. Considering this and that the two dominant harmonics $m=9$ and $m=10$ are comparable in amplitude, it is reasonable to identify the mode as a TAE. 


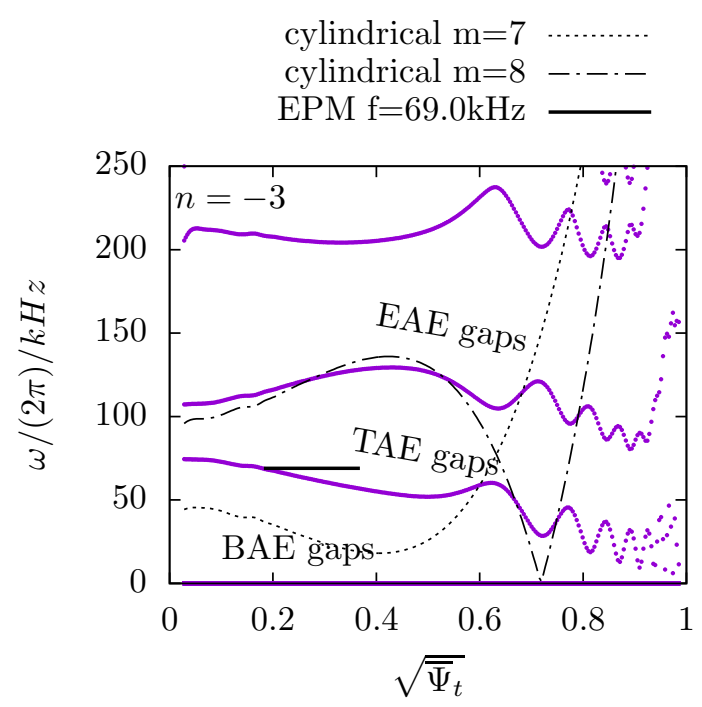

Figure 9. $n=-3$ Alfvén continua with the frequency and the HHFW of the $n=-3$ mode plotted on. Also plotted are the $m=7$ and $m=8$ Alfvén continua in the cylindrical geometry limit.

\section{B. Mode twist}

Figure 12 compares the two-dimensional mode structures calculated by MEGA and GTAW (an ideal MHD eigenvalue code[34]), which shows agreement with respect to the radial location and dominant poloidal harmonics of the modes. However, the $n=-4$ mode in the hybrid simulation takes a twisted structure on the poloidal plane, which is different from the results of the ideal MHD eigenvalue code. The twist is due to the radial phase variation of the eigenfunctions, which may be attributed to the non-perturbative kinetic effects of the fast ions[38-40]. By varying the stored energy of fast ions to change the fast ions drive in the simulations, we examined the dependence of the radial phase difference of the $n=-4$ TAE on the fast ions drive, which is shown in Fig. 13a. The radial phase difference is defined to be the phase 

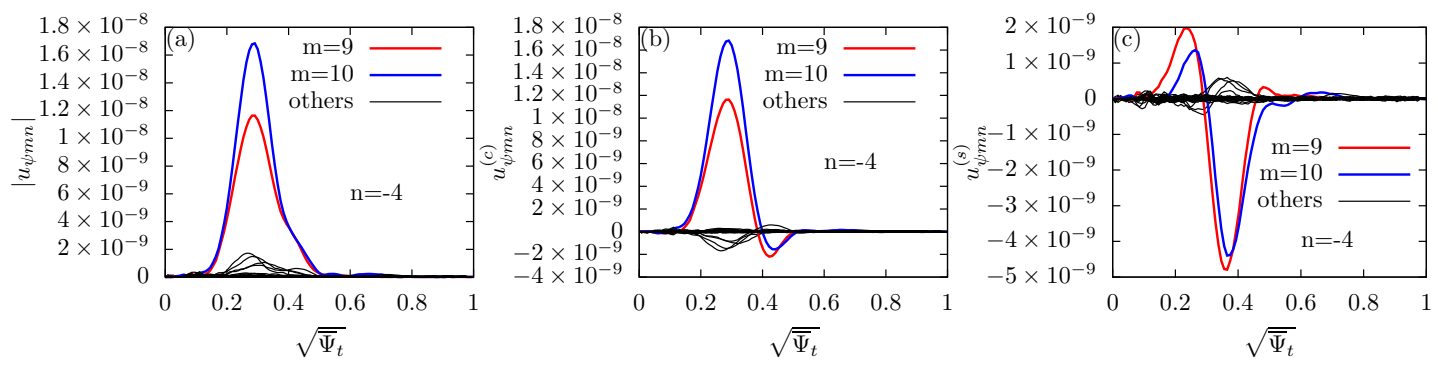

Figure 10. The radial profiles of (a) the amplitude $\left|u_{\psi m n}\right|$, (b) cosine components $u_{\psi m n}^{(c)}$, and (c) sine components $u_{\psi m n}^{(s)}$ of the $n=-4$ mode. The time for plotting these figures is chosen to be at the moment when the sine component of the dominant $m=10$ harmonic reaches zero at the radial location where the amplitude of the mode is maximal (i.e., $\sqrt{\bar{\Psi}_{t}}=0.29$ for this case). The poloidal mode number range for this case is $0 \leqslant m \leqslant 64$.

difference of the $m=10$ harmonic between the radial location $\sqrt{\bar{\Psi}_{t}}=0.173$ and $\sqrt{\bar{\Psi}_{t}}=0.356$, which is the radial range where the mode amplitude is significant, as is shown in Fig. 14. Figure 13b shows that the growth rate (indication of the fast ions drive) increases with the increasing of the stored energy of fast ions while the frequency remains the same. Figure 13a shows that the phase difference increases with the increasing of the stored energy of fast ions, i.e., the mode twist is positively correlated with the fast ions drive.

\section{SUMMARY}

Kinetic-MHD hybrid simulations are carried out to investigate possible fast-iondriven modes on the EAST tokamak. Toroidicity-induced Alfvén eigenmodes, reversed shear Alfvén eigenmodes, and energetic-particle continuum modes are observed simultaneously in the simulations. The slow-sound approximation of the Alfvén continua proves to be useful in identifying the modes found in the simula- 


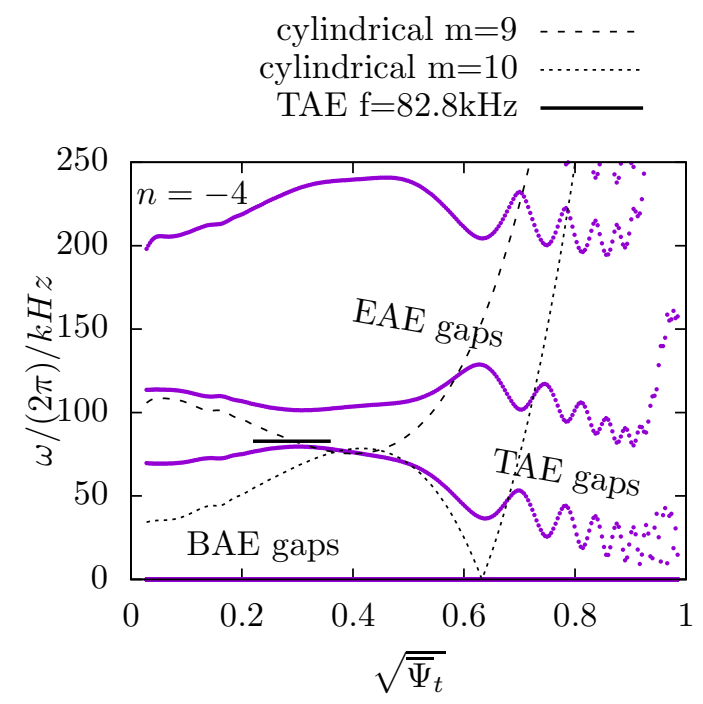

Figure 11. $n=-4$ Alfvén continua with the frequency and the HHFW of the $n=-4$ mode plotted on. Also plotted on (a) are the $m=9$ and $m=10$ Alfvén continua in the cylindrical geometry limit.

tions. The agreement between the hybrid simulations and linear eigenvalue analysis provides confidence in the simulation results. It is demonstrated numerically that the radial phase variation of the toroidicity-induced Alfvén eigenmodes is positively correlated with the fast ion drive in the hybrid simulations. The present work is limited to the linear properties of the modes and does not provide any analysis for the resonant interaction between the fast ions and the modes. This subject will be investigated in future by examining the phase space structure of the fast ion distribution function. 

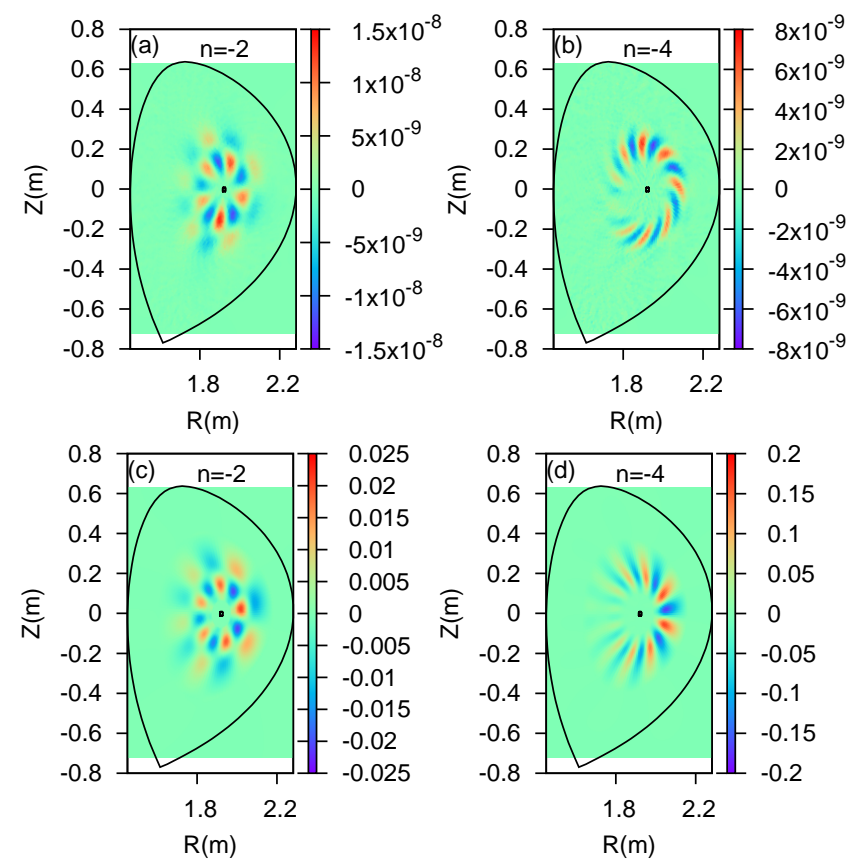

Figure 12. Contour of the radial fluid velocity on the poloidal plane calculated by MEGA ((a) and (b)) and GTAW ((c) and (d)). The $n=-2$ mode is an RSAE with frequency $f=69.0 \mathrm{kHZ}(67.9 \mathrm{kHz}$ given by GTAW). The $n=-4$ mode is an TAE with frequency $f=82 \mathrm{kHz}(83 \mathrm{kHz}$ given by GTAW). The initial stored energy of fast ions used in the MEGA simulations is $36 \mathrm{~kJ}$.

\section{ACKNOWLEDGMENTS}

One of the authors (Y. Hu) acknowledges useful discussions with H. Wang, Shaojie Wang, W. Chen, H. S. Xie, W. Shen, J. Zhu, and Sheng Wang. Numerical computations were performed on the ShenMa High Performance Computing Cluster in Institute of Plasma Physics, Chinese Academy of Sciences. This work was partially supported by the JSPS-NRF-NSFC A3 Foresight Program in the field of Plasma Physics (NSFC No. 11261140328) and the National Magnetic Confinement 

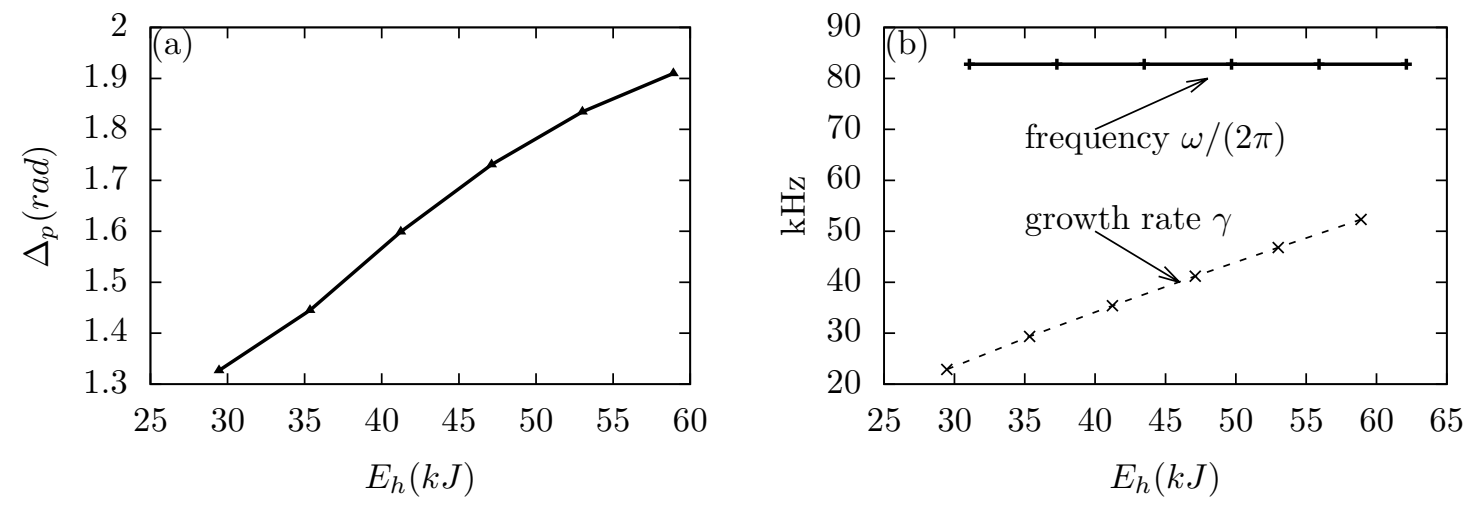

Figure 13. The dependence of (a) the phase difference, (b) mode frequency, and (b) growth rate on the initial stored energy of fast ions for the $n=-4$ TAE mode. The phase difference is defined as the phase difference of the $m=10$ harmonic between the radial location $\sqrt{\bar{\Psi}_{t}}=0.173$ and $\sqrt{\bar{\Psi}_{t}}=0.356$, as is shown in Fig. 14 .

Fusion Science Program of China under Grant Nos. 2013GB112010, 2015GB110001, and 2015GB110005.

[1] C. Z. Cheng, L. Chen, and M. S. Chance, Ann. Phys. (NY) 16121 (1984).

[2] G. Y. Fu and J. W. Van Dam, Phys. Fluids B 1, 1949 (1989).

[3] K. L. Wong, R. J. Fonck, S. F. Paul, D. R. Roberts, E. D. Fredrickson, R. Nazikian, H. K. Park, M. Bell, N. L. Bretz, R. Budny, S. Cohen, G. W. Hammett, F. C. Jobes, D. M. Meade, S. S. Medley, D. Mueller, Y. Nagayama, D. K. Owens, and E. J. Synakowski, Phys. Rev. Lett. 66, 1874 (1991).

[4] W. Heidbrink, E. Strait, E. Doyle, G. Sager, and R. Snider, Nucl. Fusion 31, 1635 (1991).

[5] W. W. Heidbrink, Plasma Phys. Controlled Fusion 37, 937 (1995). 


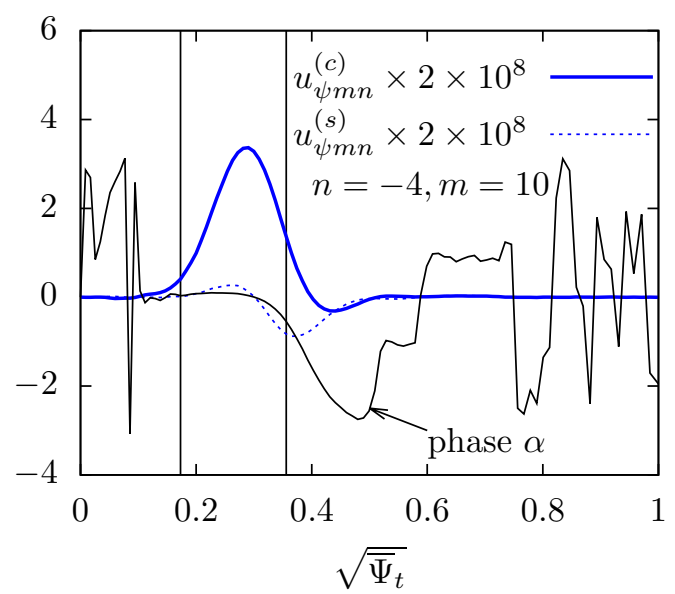

Figure 14. The radial profiles of the radial phase, cosine components $u_{\psi m n}^{(c)}$, and sine components $u_{\psi m n}^{(s)}$ of the dominant $m=10$ harmonic of the $n=-4$ TAE. The two vertical lines indicate the radial region used in calculating the phase difference given in Fig. 13.

[6] R. Nazikian, G. Y. Fu, Z. Chang, S. H. Batha, H. Berk, R. V. Budny, Y. Chen, C. Z. Cheng, D. S. Darrow, N. N. Gorelenkov, F. M. Levinton, S. Medley, M. P. Petrov, M. Redi, E. Ruskov, D. A. Spong, R. B. White, and S. J. Zweben, Phys. Plasmas 5, 1703 (1998).

[7] Y. Kusama, H. Kimura, T. Ozeki, M. Saigusa, G. J. Kramer, T. Oikawa, S. Moriyama, M. Nemoto, T. Fujita, K. Tobita, G. Y. Fu, R. Nazikian, and C. Z. Cheng, Nucl. Fusion 38, 1215 (1998).

[8] S. E. Sharapov, D. Testa, B. Alper, D. N. Borba, A. Fasoli, N. C. Hawkes, R. F. Heeter, M. Mantsinen, M. G. V. Hellermann, and contributors to the EFDA-JET work programme, Phys. Lett. A 289, 127 (2001).

[9] W. W. Heidbrink, N. N. Gorelenkov, Y. Luo, M. A. Van Zeeland, R. B. White, M. E. Austin, K. H. Burrell, G. J. Kramer, M. A. Makowski, G. R. McKee, and R. Nazikian, Phys. Rev. Lett. 99, 245002 (2007). 
[10] M. A. Van Zeeland, W. W. Heidbrink, R. K. Fisher, M. Garc a Mu oz, G. J. Kramer, D. C. Pace, R. B. White, S. Aekaeslompolo, M. E. Austin, J. E. Boom, I. G. J. Classen, S. da Gra a, B. Geiger, M. Gorelenkova, N. N. Gorelenkov, A. W. Hyatt, N. Luhmann, M. Maraschek, G. R. McKee, R. A. Moyer, C. M. Muscatello, R. Nazikian, H. Park, S. Sharapov, W. Suttrop, G. Tardini, B. J. Tobias, Y. B. Zhu, DIII-D, and A. U. Teams, Phys. Plasmas 18, 056114 (2011).

[11] M. V. Zeeland, N. Gorelenkov, W. Heidbrink, G. Kramer, D. Spong, M. Austin, R. Fisher, M. G. Munoz, M. Gorelenkova, N. Luhmann, M. Murakami, R. Nazikian, D. Pace, J. Park, B. Tobias, and R. White, Nucl. Fusion 52, 094023 (2012).

[12] Y. Todo, H. L. Berk, and B. N. Breizman, Phys. Plasmas 10, 2888 (2003).

[13] S. Pinches, V. Kiptily, S. Sharapov, D. Darrow, L.-G. Eriksson, H.-U. Fahrbach, M. García-Muñoz, M. Reich, E. Strumberger, A. Werner, the ASDEX Upgrade Team, and J.-E. Contributors, Nucl. Fusion 46, S904 (2006).

[14] R. B. White, E. Fredrickson, D. Darrow, M. Zarnstorff, R. Wilson, S. Zweben, K. Hill, Y. Chen, and G. Fu, Phys. Plasmas 2, 2871 (1995).

[15] B. N. Breizman and S. E. Sharapov, Plasma Phys. Controlled Fusion 53, 054001 (2011).

[16] N. Gorelenkov, S. Pinches, and K. Toi, Nucl. Fusion 54, 125001 (2014).

[17] R. Zhang, G. Fu, R. White, and X. Wang, Nucl. Fusion 55, 122002 (2015).

[18] B. Wan, J. Li, H. Guo, Y. Liang, G. Xu, L. Wang, X. Gong, and A. Garofalo for the EAST Team and Collaborators, Nucl. Fusion 55, 104015 (2015).

[19] Y. Todo and T. Sato, Phys. Plasmas 5, 1321 (1998).

[20] Y. Todo, Phys. Plasmas (1994-present) 13, (2006).

[21] Y. Todo, M. V. Zeeland, A. Bierwage, and W. Heidbrink, Nucl. Fusion 54, 104012 (2014).

[22] J. Zhu, Z. W. Ma, and G. Y. Fu, Phys. Plasmas 20, 122508 (2013). 
[23] S. E. Sharapov, B. Alper, H. L. Berk, D. N. Borba, B. N. Breizman, C. D. Challis, A. Fasoli, N. C. Hawkes, T. C. Hender, J. Mailloux, S. D. Pinches, D. Testa, and EFDA JET work programme, Phys. Plasmas 9, 2027 (2002).

[24] A. Fukuyama and T. Akutsu, Lyon, 2002, Proc. 19th Int. Conf. on Fusion Energy, Vienna: IAEA CD-ROM file TH/P3-14.

[25] B. N. Breizman, H. L. Berk, M. S. Pekker, S. D. Pinches, and S. E. Sharapov, Phys. Plasmas 10, 3649 (2003).

[26] G. Y. Fu and H. L. Berk, Phys. Plasmas 13, 052502 (2006).

[27] H. Wang and Y. Todo, Journal of the Physical Society of Japan 80, 094501 (2011).

[28] Y. Chen, T. Munsat, S. E. Parker, W. W. Heidbrink, M. A. Van Zeeland, B. J. Tobias, and C. W. Domier, Phys. Plasmas 20, 012109 (2013).

[29] W. Chen, L. Yu, Y. Liu, X. Ding, H. Xie, J. Zhu, L. Yu, X. Ji, J. Li, Y. Li, D. Yu, Z. Shi, X. Song, J. Cao, S. Song, Y. Dong, W. Zhong, M. Jiang, Z. Cui, Y. Huang, Y. Zhou, J. Dong, M. Xu, F. Xia, L. Yan, Q. Yang, X. Duan, and the HL-2A Team, Nucl. Fusion 54, 104002 (2014).

[30] H. Xie and Y. Xiao, Phys. Plasmas 22, 022518 (2015).

[31] D. Spong, Nucl. Fusion 53, 053008 (2013).

[32] L. Chen, Phys. Plasmas 1, 1519 (1994).

[33] L. Chen and F. Zonca, Nucl. Fusion 47, S727 (2007).

[34] Y. Hu, G. Li, N. N. Gorelenkov, H. Cai, W. Yang, D. Zhou, and Q. Ren, Phys. Plasmas 21, 052510 (2014).

[35] B. Tobias, E. Bass, I. Classen, C. Domier, B. Grierson, W. Heidbrink, N. L. Jr, R. Nazikian, H. Park, D. Spong, and M. V. Zeeland, Nucl. Fusion 52, 103009 (2012).

[36] B. J. Tobias, I. G. J. Classen, C. W. Domier, W. W. Heidbrink, N. C. Luhmann, R. Nazikian, H. K. Park, D. A. Spong, and M. A. Van Zeeland, Phys. Rev. Lett. 106, 075003 (2011). 
[37] W. W. Heidbrink, M. E. Austin, D. A. Spong, B. J. Tobias, and M. A. Van Zeeland, Phys. Plasmas 20, 082504 (2013).

[38] Z. Wang, Z. Lin, I. Holod, W. W. Heidbrink, B. Tobias, M. V. Zeeland, and M. E. Austin, Phys. Rev. Lett 111, 145003 (2013).

[39] W. Zhang, I. Holod, Z. Lin, and Y. Xiao, Phys. Plasmas 19, 022507 (2012).

[40] R. Ma, F. Zonca, and L. Chen, Phys. Plasmas 22, 092501 (2015).

[41] L. Lao, H. S. John, R. Stambaugh, A. Kellman, and W. Pfeiffer, Nucl. Fusion 25, $1611(1985)$.

[42] Iter physics basis editors, Nucl. Fusion 39, 2471 (1999).

[43] Y. Todo, AIP Conference Proceedings 1478, 141 (2012).

[44] M. S. Chu, J. M. Greene, L. L. Lao, A. D. Turnbull, and M. S. Chance, Phys. Fluids B 4, 3713 (1992).

[45] W. Deng, Z. Lin, I. Holod, Z. Wang, Y. Xiao, and H. Zhang, Nucl. Fusion 52, 043006 (2012). 